\title{
Quantitative Self-Sensitized Photooxidation of 1,2-Diarylcyclobutene Derivatives by Singlet Oxygen
}

\author{
Joël Kühni, Jean-Luc Debieux, Peter Belser* \\ Department of Chemistry, University of Fribourg, Chemin du Musée 9, 1700 Fribourg, Switzerland \\ Fax +41(26)3009738; E-mail: Peter.Belser@unifr.ch \\ Received 27 February 2007
}

\begin{abstract}
Ligands 1 and $\mathbf{2}$ underwent quantitative photooxidation when they were reacted with singlet oxygen sensitized by methylene blue. However, the quantitative reaction became self-sensitized for the compound $\mathbf{R u}(\mathbf{1})$ wherein the ruthenium complex acted as a sensitizer.
\end{abstract}

Key words: complexes, heterocycles, ligands, photooxidation, ruthenium

The oxidation of olefins to alcohols or ketones by singlet oxygen has been extensively reported. ${ }^{1}$ In this type of reaction, singlet oxygen is produced in situ by a photosensitization process that consists of an energy transfer from the excited state of a sensitizer to the triplet ground state of $\mathrm{O}_{2}\left({ }^{3} \Sigma_{\mathrm{g}}{ }^{-}\right){ }^{2}$ Organic molecules as well as coordination compounds like methylene blue $\mathrm{e}^{3-5}$ or ruthenium complexes $^{6}$, respectively, are usually used as photosensitizers. However, the reaction becomes self-sensitized if the substrate acts as its own sensitizer. ${ }^{7}$ This principle has been employed in the photodynamic treatment of malignant tumors. ${ }^{8}$

Photooxidation reaction between 1,2-diphenylcyclobutene and singlet oxygen can take place in three different modes $^{5,9}$ (Scheme 1). The first involves a [2+2] cycloaddition reaction between singlet oxygen and the double bond in the cyclobutene moiety. The resulting dioxetane A is easily cleaved either thermally or photochemically to form the diketone $\mathbf{B}$. The second type of photooxidation is a [4+2] cycloaddition reaction producing the endoperoxide molecule $\mathbf{C}$ which rearranges either to dioxetane $\mathbf{A}$ to yield $\mathbf{B}$ or to phenol-epoxide $\mathbf{D}$ to form $\mathbf{E}$ (Scheme 1). The last type of photooxidation process requires an allylic hydrogen to perform a [3+2] cycloaddition reaction to obtain the allylic hydroperoxide $\mathbf{F}$.

Previously reported photooxidation reactions of 1,2-diarylcyclobutene derivatives with singlet oxygen yielded the product with two-carbonyl functions ( $\mathbf{B}$ in the case of 1,2-diphenylcyclobutene) in poor to moderate yields (14$40 \%)^{3,5,9}$

Here, we report quantitative photooxidation reactions of non-coordinated ligands $\mathbf{1}$ and $\mathbf{2}$ (Scheme 2) sensitized by methylene blue and also, a self-sensitized reaction involving the transformation of the coordinated ligands in complex Ru-1 (Schemes 3 and 5) to give products with two ketone functionalities (Schemes 4 and 5). These quantitative yields can be explained as follows. Firstly, ligands 1 and $\mathbf{2}$ and complex Ru-1 rearrange exclusively to bis-ketone compounds after the [4+2] cycloaddition reaction be-



Scheme 1 Different mechanisms of photooxidation reaction between singlet oxygen and 1,2-diphenylcyclobutene

SYNTHESIS 2007, No. 9, pp 1421-1425

Advanced online publication: 10.04 .2007

DOI: 10.1055/s-2007-966007; Art ID: Z05307SS

(c) Georg Thieme Verlag Stuttgart · New York 
cause of the absence of protons at position 2 in the thiophene moieties. Secondly, ligands $\mathbf{1}$ and $\mathbf{2}$ as well as complex Ru-1 do not have allylic protons at positions 3 and 4 in the cyclobutene fragment. Therefore, the $[2+3]$ cycloaddition reaction cannot take place and hence, the allylic hydroperoxide derivatives are not formed. Additionally, singlet oxygen can react with ligands $\mathbf{1}$ and $\mathbf{2}$ and also with complex Ru-1. For both forms of the ligands, noncoordinated and complexed, either [2+2] or [4+2] cycloaddition reaction is accessible. In both cases, compounds with two carbonyl functions are obtained.

Ligand 1 was synthesized in a one-step reaction by double condensation between $\mathbf{3}^{10}$ and $\mathbf{4}^{11}$ (Scheme 2).

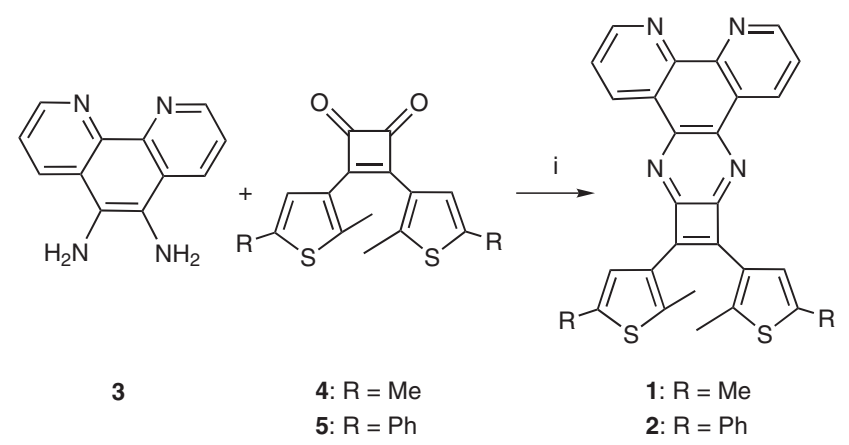

Scheme 2 Reagents and conditions: i) Dean-Stark assembly, toluene-AcOH 5:1, reflux, $72 \mathrm{~h}$.

We have optimized the boundary conditions for the synthesis of ligand 1 (Table 1). By refluxing in ethanol (entry 1 ), methanol (entry 3 ) or acetic acid (entry 4), only traces of ligand 1 were detected. By heating at $400-500{ }^{\circ} \mathrm{C}$ without any solvent, 4 decomposed completely (entry 2 ). In deaerated acetic acid at boiling point, ligand $\mathbf{1}$ was formed in 5\% yield (entry 5). With a Dean-Stark assembly to remove water, ligand $\mathbf{1}$ was formed in $13 \%$ yield in deaerated toluene-acetic acid (15:2) (entry 6) and $29 \%$ yield in deaerated toluene-acetic acid 5:1 (entry 7); both with a reaction time of 24 hours. Finally, we found the best conditions in deaerated toluene-acetic acid $(5: 1)$ with a reaction time of 72 hours (entry 8 ) giving $32 \%$ yield.

Ligand 2 was synthesized under the same conditions but the double condensation reaction occurring between $3^{10}$ and $\mathbf{5}^{12}$ gave a moderate yield of $22 \%$.

The synthesis of the metal complex Ru-1 (Scheme 3) was performed under argon in an AtmosBag TM $_{\text {(Aldrich: }}$ $\mathrm{Z} 112828$-1EA). $\mathrm{Ru}(\mathrm{bpy})_{2} \mathrm{Cl}_{2} \cdot 2 \mathrm{H}_{2} \mathrm{O}$ and ligand $\mathbf{1}$ were heated under reflux in ethanol-water (3:1) overnight. Solvents were removed and the residue was dissolved in water. The complex Ru-1 was precipitated with $\mathrm{NH}_{4} \mathrm{PF}_{6}$ and purified on a silica gel preparative plate. $\mathrm{Ru}-\mathbf{1}$ was obtained in a yield of 58\%. Any kind of manipulation will have to be carried out under oxygen free atmosphere to avoid the photooxidation of $\mathrm{Ru}-\mathbf{1}$ to $\mathrm{Ru}-\mathbf{1}(\mathrm{O})$ (Scheme 5). This complexation reaction was also performed in solvents like methoxyethanol or 1,2-dichloromethane/water

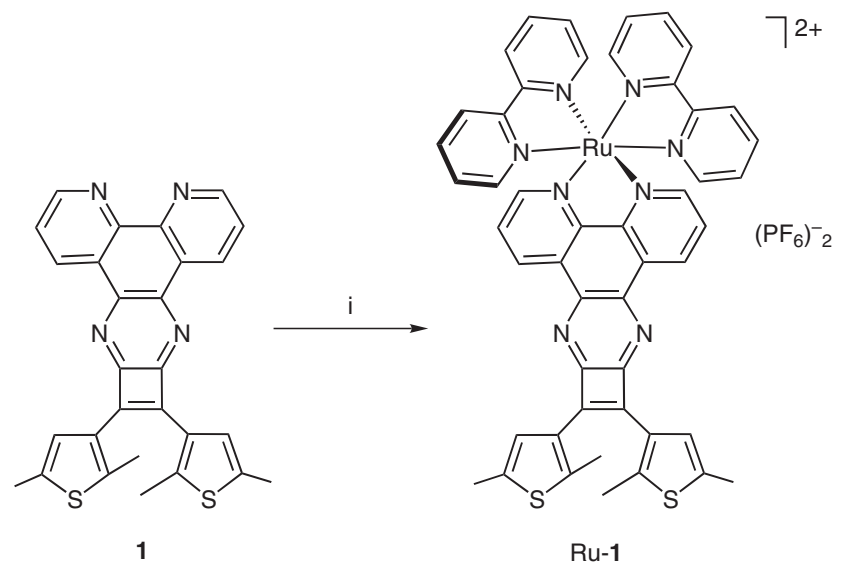

Scheme 3 Reagents and conditions: i) $\mathrm{Ru}(\mathrm{bpy})_{2} \mathrm{Cl}_{2} \cdot 2 \mathrm{H}_{2} \mathrm{O}$, EtOH$\mathrm{H}_{2} \mathrm{O}$ (3:1), argon atmosphere, reflux, $24 \mathrm{~h}$.

Table 1 Double Condensation Reaction of 5,6-Diaminophenanthroline (3) ${ }^{10}$ with 3,4-Bis $(2,5 \text {-dimethylthien-3-yl)cyclobutenedione (4) })^{11}$ (Scheme 2)

\begin{tabular}{|c|c|c|c|c|c|c|c|c|}
\hline Entry & $\begin{array}{l}\mathbf{3} \\
(\mathrm{mmol})\end{array}$ & $\begin{array}{l}4 \\
\text { (equiv) }\end{array}$ & $\begin{array}{l}\text { Solvent } \\
(\mathrm{mL})\end{array}$ & $\begin{array}{l}\text { Acetic acid } \\
(\mathrm{mL})\end{array}$ & $\begin{array}{l}\text { Temp } \\
\left({ }^{\circ} \mathrm{C}\right)\end{array}$ & $\begin{array}{l}\text { Time } \\
\text { (h) }\end{array}$ & $\begin{array}{l}\text { Recovered } 4 \\
(\%)\end{array}$ & $\begin{array}{l}\text { Yield } \\
(\%)\end{array}$ \\
\hline 1 & 1.8 & 0.66 & EtOH (40) & 0.4 & 79 & 22 & 79 & traces \\
\hline 2 & 0.1 & 1 & - & - & $400-500$ & 0.25 & 0 & 0 \\
\hline $3^{a}$ & 0.5 & 1 & $\mathrm{MeOH}(15)$ & 0.15 & 65 & 17 & 86 & traces \\
\hline $4^{a}$ & 1.5 & 0.9 & $\mathrm{AcOH}$ & 40 & 118 & 24 & 87 & traces \\
\hline $5^{c}$ & 0.5 & 1 & $\mathrm{AcOH}$ & 10 & 118 & 24 & 66 & 5 \\
\hline $6^{b, c}$ & 0.5 & 1 & toluene (15) & 2 & 111 & 24 & 53 & 13 \\
\hline $7^{b, c}$ & 0.6 & 1 & toluene (10) & 2 & 111 & 24 & 55 & 29 \\
\hline $8^{\mathrm{b}, \mathrm{c}}$ & 1.2 & 1 & toluene (20) & 4 & 111 & 72 & 47 & 32 \\
\hline
\end{tabular}

a Addition of $\mathrm{MgSO}_{4}$.

${ }^{\mathrm{b}}$ Dean-Stark assembly to remove water was used.

${ }^{\mathrm{c}}$ Argon was bubbled for $20 \mathrm{~min}$. 

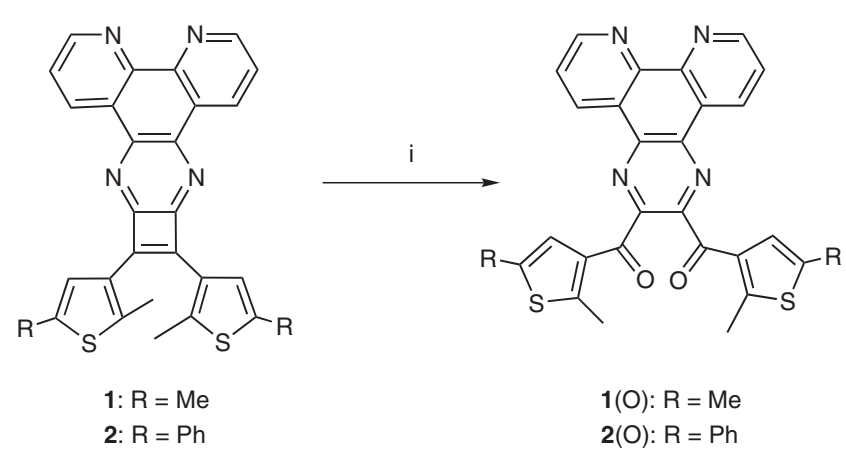

Scheme 4 Reagents and conditions: i) Oxygen bubbling in deuterated chloroform with methylene blue as photosensitizer; irradiation with a $300 \mathrm{~W}$ lamp.

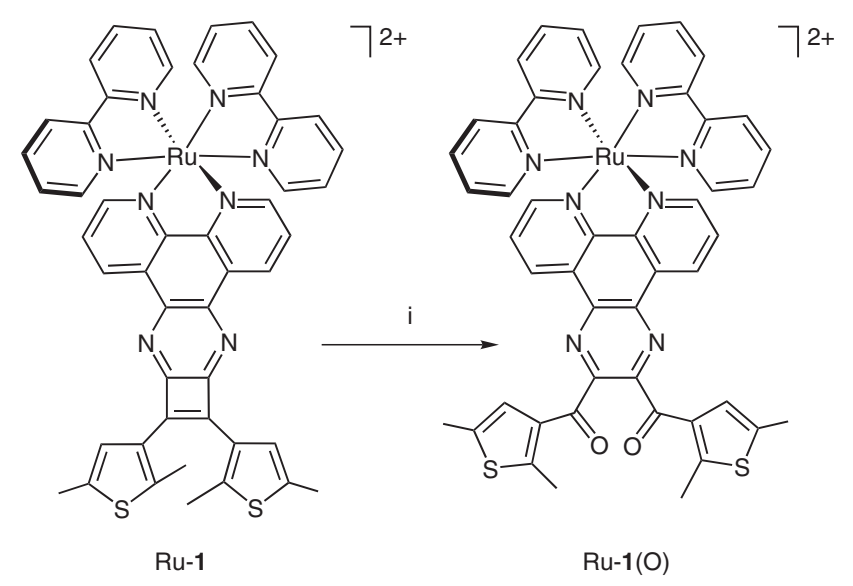

Scheme 5 Reagents and conditions: i) Oxygen bubbling in deuterated acetonitrile; irradiation with a $300 \mathrm{~W}$ lamp.

(1:1) but were unsuccessful due to the decomposition of ligand 1 to unknown products under such conditions.

Photooxidation of ligands $\mathbf{1}$ and $\mathbf{2}$ were performed in the same way (Scheme 4). In an NMR tube, the ligand was dissolved in deuterated chloroform containing methylene blue as photosensitizer. The reaction mixture was irradiated under oxygen bubbling with a $300 \mathrm{~W}$ incandescent lamp for 3 hours. Methylene blue was removed by extraction. Ligands $\mathbf{1}(\mathrm{O})$ and $\mathbf{2}(\mathrm{O})$ were obtained pure and hence isolated in quantitative yield without any purification.

Photooxidation of complex Ru-1 was performed in deuterated acetonitrile (Scheme 5) in an NMR tube. The reaction mixture was irradiated under oxygen bubbling with a $300 \mathrm{~W}$ incandescent lamp for 15 minutes. The solvent was removed to give complex $\mathrm{Ru}-\mathbf{1}(\mathrm{O})$ and isolated in quantitative yield without any purification.

Analytical data are consistent with the given structures of photooxidized products. The number of peaks in ${ }^{1} \mathrm{H}$ NMR and ${ }^{13} \mathrm{C}$ NMR before and after photooxidation were identical meaning the symmetry of the compounds did not change during the photooxidation process. Mass spectrum and high-resolution mass spectrum of photooxidized products showed an increase in molecular weight by $32 \mathrm{~g} /$ mol corresponding to the incorporation of two atoms of oxygen. A new band around $1650 \mathrm{~cm}^{-1}$ in the IR spectrum allows an attribution of these oxygen atoms to carbonyl functions.

To conclude, we have developed synthetic methods involving a double condensation reaction to get ligands $\mathbf{1}$ and 2. Ligand $\mathbf{1}$ was coordinated to a ruthenium center giving Ru-1 under mild conditions. We have performed quantitative photooxidation reactions of ligands $\mathbf{1}$ and $\mathbf{2}$ with singlet oxygen, produced by a photosensitized process using methylene blue as sensitizer, as well as a selfsensitized photooxidation reaction for complex $\mathrm{Ru}-\mathbf{1}$.

Column chromatography was performed using silica gel (230-400 or 400-600 mesh size) from Chemie Brunschweig AG. Preparative plates $(20 \times 20 \mathrm{~cm})$ with silica gel $60 \mathrm{~F}_{254}$, having a layer thickness of $2 \mathrm{~mm}$ (for preparative plate chromatography) and aluminum sheets coated with silica gel $60 \mathrm{~F}_{254}$ (for TLC) were purchased from Merck. A mixture of $\mathrm{MeCN}, \mathrm{MeOH}$ and $10 \%$ aq $\mathrm{KNO}_{3}$ in a volume ratio of 40:10:1 respectively, was used as the eluent. All products were characterized by ${ }^{1} \mathrm{H}$ NMR and ${ }^{13} \mathrm{C}$ NMR, on Bruker Avance DRX-400 (400.13 MHz, for ${ }^{1} \mathrm{H}$ NMR and $100.62 \mathrm{MHz}$ for ${ }^{13} \mathrm{C}$ NMR) and on Bruker Avance DRX-360 (360 MHz, for ${ }^{1} \mathrm{H}$ NMR and $90.55 \mathrm{MHz}$ for ${ }^{13} \mathrm{C}$ NMR spectrometer). Chemical shifts are given in ppm using the solvent itself as internal standard. The chemical shifts are expressed as $\delta$ values and the coupling constants $(J)$ are given in Hertz. Mass spectra were recorded on HP 5988A Quadrupole (EI ionization, $70 \mathrm{eV}$ ) mass spectrometer. ESI and high-resolution mass spectra were recorded on a Bruker FTMS 4.7T BioAPEXII spectrometer. GC-MS analyses were done on ThermeQuest Finnigan VOYAGER GS/MS Trace GC 2000 Series equipped with an Optima-5-MS column $(0.25 \mu \mathrm{m}, 25 \mathrm{~m} \times 0.32 \mathrm{~mm}$; Marcherey-Nagel). UV/vis spectra were recorded with a PerkinElmer Lambda 40. The wavelength maxima are reported in $\mathrm{nm}$.

\section{Ligand 1}

In a Dean-Stark assembly, the corresponding 1,2-diketone 4 (362 mg, $1.2 \mathrm{mmol}, 1.0$ equiv) and phenanthroline-5,6-diamine 3 ( $252 \mathrm{mg}, 1.2 \mathrm{mmol}, 1.0$ equiv) were dissolved in deaerated toluene $(20 \mathrm{~mL})$ and $\mathrm{AcOH}(4 \mathrm{~mL})$ under argon. The mixture was stirred for $24 \mathrm{~h}$ under reflux $\left(130^{\circ} \mathrm{C}\right)$. Solvents were removed by distillation. The residue was chromatographed on silica gel using $\mathrm{CH}_{2} \mathrm{Cl}_{2}-\mathrm{Et}_{3} \mathrm{~N}$ (100:1) as eluent to give 1; yield: $182 \mathrm{mg}$ (32\%).

IR (KBr): 2914, 2852, 1664, 1522, 1484, 1430, 1364, 1298, 1214, $1188,1144,922,804,736 \mathrm{~cm}^{-1}$.

${ }^{1} \mathrm{H} \mathrm{NMR}\left(\mathrm{CDCl}_{3}\right): \delta=2.44(\mathrm{~s}, 6 \mathrm{H}), 2.84(\mathrm{~s}, 6 \mathrm{H}), 6.88(\mathrm{~s}, 2 \mathrm{H}), 7.66$ (dd, $J=4.3,8.4 \mathrm{~Hz}, 2 \mathrm{H}), 9.14$ (dd, $J=4.3,1.6 \mathrm{~Hz}, 2 \mathrm{H}), 9.24$ (dd, $J=1.8,8.2 \mathrm{~Hz}, 2 \mathrm{H})$.

${ }^{13} \mathrm{C} \mathrm{NMR}\left(\mathrm{CDCl}_{3}\right): \delta=174.2,153.1,146.5,144.0,137.7,135.9$, 135.1, 131.9, 125.6, 123.1, 15.8, 14.9.

MS (EI): $m / z=477.12\left(\mathrm{M}^{+}+1\right)$.

HRMS: $m / z$ calcd for $\mathrm{C}_{28} \mathrm{H}_{21} \mathrm{~N}_{4} \mathrm{~S}_{2}\left(\mathrm{M}^{+}+1\right)$ : 477.1202; found: 477.1197.

UV/vis $\quad\left(\mathrm{CHCl}_{3}\right): \quad \lambda_{\max }(\varepsilon)=288 \quad(30700), \quad 315 \quad(33900), 450$ $\mathrm{nm}(3400)$.

\section{Ligand 2}

In a Dean-Stark assembly, the corresponding 1,2-diketone 5 (511 mg, $1.2 \mathrm{mmol}, 1.0$ equiv) and phenanthroline-5,6-diamine $\mathbf{3}$ (252 mg, $1.2 \mathrm{mmol}, 1.0$ equiv) were dissolved in deaerated toluene $(20 \mathrm{~mL})$ and $\mathrm{AcOH}(4 \mathrm{~mL})$ under argon. The mixture was stirred for $24 \mathrm{~h}$ under reflux $\left(130^{\circ} \mathrm{C}\right)$. Solvents were removed by distillation. 
The residue was chromatographed on silica gel using $\mathrm{CH}_{2} \mathrm{Cl}_{2}-\mathrm{Et}_{3} \mathrm{~N}$ (100:1) as eluent to give 2; yield: $160 \mathrm{mg}(22 \%)$.

IR (KBr): 3056, 3020, 2960, 2914, 2848, 1670, 1540, 1486, 1366, $1296,1262,1190,1092,1018,804,738,686 \mathrm{~cm}^{-1}$.

${ }^{1} \mathrm{H} \mathrm{NMR}\left(\mathrm{CDCl}_{3}\right): \delta=2.98(\mathrm{~s}, 6 \mathrm{H}), 7.31(\mathrm{tt}, J=7.33,1.52 \mathrm{~Hz}, 2$ $\mathrm{H}), 7.37(\mathrm{tt}, J=7.33,1.52 \mathrm{~Hz}, 4 \mathrm{H}), 7.53-7.57(\mathrm{~m}, 6 \mathrm{H}), 7.74(\mathrm{dd}$, $J=8.21,4.42 \mathrm{~Hz}, 2 \mathrm{H}), 9.24(\mathrm{dd}, J=4.29,1.26 \mathrm{~Hz}, 2 \mathrm{H}), 9.31(\mathrm{dd}$, $J=8.21,1.64 \mathrm{~Hz}, 2 \mathrm{H})$.

${ }^{13} \mathrm{C} \mathrm{NMR}\left(\mathrm{CDCl}_{3}\right): \delta=174.8,153.4,149.9,146.2,142.6,135.8$, 133.9, 133.5, 129.4, 129.3, 129.1, 128.4, 128.1, 125.8, 123.9, 122.8, 16.4 .

MS (EI): $m / z=601.16\left(\mathrm{M}^{+}+1\right)$.

HRMS: $m / z$ calcd for $\mathrm{C}_{38} \mathrm{H}_{25} \mathrm{~N}_{4} \mathrm{~S}_{2}(\mathrm{M}+1)$ : 601.1515; found: 601.1524.

UV/Vis $\left(\mathrm{CHCl}_{3}\right): \lambda_{\max }(\varepsilon)=305$ (51900), $365(16200), 483 \mathrm{~nm}$ (3000).

\section{Metal Complex Ru-1}

The synthesis was performed under argon in an AtmosBag ${ }_{\mathrm{TM}}$ (Aldrich: Z112828-1EA). A $25 \mathrm{~mL}$, one-necked, round-bottomed flask was charged with the ligand $1(20 \mathrm{mg}, 44.2 \mu \mathrm{mol}, 1$ equiv), $\mathrm{Ru}(\mathrm{bpy})_{2} \mathrm{Cl}_{2} \cdot 2 \mathrm{H}_{2} \mathrm{O}(25 \mathrm{mg}, 48.6 \mu \mathrm{mol}, 1.1$ equiv), EtOH $(15 \mathrm{~mL})$, and $\mathrm{H}_{2} \mathrm{O}(5 \mathrm{~mL})$. The solution was refluxed overnight under argon. The organic phase was extracted with $\mathrm{H}_{2} \mathrm{O}(3 \times 20 \mathrm{~mL})$ and the aqueous phase was washed with $\mathrm{CH}_{2} \mathrm{Cl}_{2}(3 \times 20 \mathrm{~mL})$. The residual $\mathrm{CH}_{2} \mathrm{Cl}_{2}$ in the aqueous phase was removed in vacuo followed by the addition of $\mathrm{NH}_{4} \mathrm{PF}_{6}(0.5 \mathrm{~g})$. The precipitate, thus formed, was collected by filtration and purified on a silica gel plate $(\mathrm{MeCN}-$ MeOH-10\% aq $\left.\mathrm{KNO}_{3}, 40: 10: 1\right)$ to afford Ru-1; yield: $30 \mathrm{mg}$ $(58 \%)$.

IR (KBr): 3076, 2917, 2851, 1603, 1526, 1465, 1446, 1370, 839, $762,730 \mathrm{~cm}^{-1}$.

${ }^{1} \mathrm{H}$ NMR $\left(\mathrm{CD}_{3} \mathrm{CN}\right): \delta=2.39(\mathrm{~s}, 6 \mathrm{H}), 2.41(\mathrm{~s}, 6 \mathrm{H}), 6.63(\mathrm{~s}, 2 \mathrm{H})$, $7.26(\mathrm{dd}, J=6.4,6.4 \mathrm{~Hz}, 2 \mathrm{H}), 7.47(\mathrm{dd}, J=6.4,6.4 \mathrm{~Hz}, 2 \mathrm{H}), 7.69$ $(\mathrm{d}, J=5.0 \mathrm{~Hz}, 2 \mathrm{H}), 7.85(\mathrm{~d}, J=5.5 \mathrm{~Hz}, 2 \mathrm{H}), 7.87(\mathrm{~d}, J=5.5 \mathrm{~Hz}, 2$ H), $8.01(\mathrm{dd}, J=7.7,7.7 \mathrm{~Hz}, 2 \mathrm{H}), 8.12(\mathrm{dd}, J=7.9,7.9 \mathrm{~Hz}, 2 \mathrm{H})$, $8.17(\mathrm{~d}, J=5.0 \mathrm{~Hz}, 2 \mathrm{H}), 8.52(\mathrm{~d}, J=8.2 \mathrm{~Hz}, 2 \mathrm{H}), 8.56(\mathrm{~d}$, $J=8.2 \mathrm{~Hz}, 2 \mathrm{H}), 9.51(\mathrm{~d}, J=8.2 \mathrm{~Hz}, 2 \mathrm{H})$.

MS (ESI): $m / z=1035.12\left(\mathrm{M}^{+}-\mathrm{PF}_{6}^{-}\right)$.

HRMS: $m / z$ calcd for $\mathrm{C}_{48} \mathrm{H}_{36} \mathrm{~F}_{6} \mathrm{~N}_{8} \mathrm{PRuS}_{2}\left(\mathrm{M}^{+}-\mathrm{PF}_{6}\right)$ : 1035.1184 ; found: 1035.1179 .

UV/vis $(\mathrm{MeCN}): \lambda_{\max }(\varepsilon)=287(87100), 420(19000), 456 \mathrm{~nm}$ (23500).

\section{Ligand 1(O)}

In an NMR tube, ligand $\mathbf{1}$ ( $4 \mathrm{mg}, 4.8 \mathrm{mmol}, 1.0$ equiv) was dissolved in $\mathrm{CHCl}_{3}(0.75 \mathrm{~mL})$ containing methylene blue $\left(10^{-4} \mathrm{M}\right)$. The mixture was irradiated under $\mathrm{O}_{2}$ bubbling with a $300 \mathrm{~W}$ lamp for $3 \mathrm{~h}$. The mixture was then poured into $\mathrm{H}_{2} \mathrm{O}(1 \mathrm{~mL})$ and extracted with $\mathrm{CH}_{2} \mathrm{Cl}_{2}(3 \times 1 \mathrm{~mL})$. The organic extract was dried $\left(\mathrm{MgSO}_{4}\right)$ and the solvent removed under vacuum to afford ligand $1(\mathrm{O})$; yield: $4 \mathrm{mg}$ (ca. 100\%).

IR (KBr): 2962, 2916, 2600, 2494, 1644, 1472, 1358, 1252, 1124, $190,742,634 \mathrm{~cm}^{-1}$.

${ }^{1} \mathrm{H} \mathrm{NMR}\left(\mathrm{CDCl}_{3}\right): \delta=2.42(\mathrm{~s}, 6 \mathrm{H}), 2.71(\mathrm{~s}, 6 \mathrm{H}), 7.10(\mathrm{~s}, 2 \mathrm{H}), 7.85$ (dd, $J=8.21,4.42 \mathrm{~Hz}, 3 \mathrm{H}), 9.37$ (dd, $J=4.55,1.77 \mathrm{~Hz}, 3 \mathrm{H}), 9.47$ (dd, $J=8.08,1.77 \mathrm{~Hz}, 2 \mathrm{H}$ ).

${ }^{13} \mathrm{C}$ NMR $\left(\mathrm{CDCl}_{3}\right): \delta=15.0,16.3,124.4,126.5,127.9,133.9$, 134.0, 135.2, 138.2, 151.4, 152.5, 152.9, 187.3.

MS (ESI): $m / z=509.1\left(\mathrm{M}^{+}+1\right)$.
HRMS: $\mathrm{m} / \mathrm{z}$ calcd for $\mathrm{C}_{28} \mathrm{H}_{21} \mathrm{~N}_{4} \mathrm{O}_{2} \mathrm{~S}_{2}\left(\mathrm{M}^{+}+1\right)$ : 509.1100; found: 509.1101 .

UV/Vis $\left(\mathrm{CHCl}_{3}\right): \lambda_{\max }(\varepsilon)=266(33300), 314(14800), 357 \mathrm{~nm}$ (7800).

\section{Ligand 2(O)}

In an NMR tube, ligand 2 (4 mg, $4.8 \mathrm{mmol}, 1.0$ equiv) was dissolved in $\mathrm{CHCl}_{3}(0.75 \mathrm{~mL})$ containing methylene blue $\left(10^{-4} \mathrm{M}\right)$. The mixture was irradiated under $\mathrm{O}_{2}$ bubbling with a $300 \mathrm{~W}$ lamp for $3 \mathrm{~h}$. Then the mixture was poured into $\mathrm{H}_{2} \mathrm{O}(1 \mathrm{~mL})$ and extracted with $\mathrm{CH}_{2} \mathrm{Cl}_{2}(3 \times 1 \mathrm{~mL})$. The organic phase was dried $\left(\mathrm{MgSO}_{4}\right)$ and the solvent removed under vacuum to afford ligand $2(\mathrm{O})$; yield: $4 \mathrm{mg}$ (ca. 100\%).

IR (KBr): 2920, 2854, 1648, 1586, 1534, 1494, 1458, 1362, 1238, $1122,1026,948,758,688 \mathrm{~cm}^{-1}$.

${ }^{1} \mathrm{H} \mathrm{NMR}\left(\mathrm{CDCl}_{3}\right): \delta=2.81(\mathrm{~s}, 6 \mathrm{H}), 7.28(\mathrm{~m}, 2 \mathrm{H}), 7.34(\mathrm{~m}, 4 \mathrm{H})$, $7.52(\mathrm{~m}, 4 \mathrm{H}), 7.68(\mathrm{~s}, 2 \mathrm{H}), 7.86(\mathrm{dd}, J=8.21,4.42 \mathrm{~Hz}, 2 \mathrm{H}), 9.40$ (dd, $J=4.55,1.77 \mathrm{~Hz}, 2 \mathrm{H}), 9.51$ (dd, $J=8.08,1.77 \mathrm{~Hz}, 2 \mathrm{H})$.

${ }^{13} \mathrm{C}$ NMR: Could not be measured owing to extreme low solubility. MS (ESI): $m / z=633.1\left(\mathrm{M}^{+}+1\right)$.

HRMS: $m / z$ calcd for $\mathrm{C}_{38} \mathrm{H}_{25} \mathrm{~N}_{4} \mathrm{O}_{2} \mathrm{~S}_{2}(\mathrm{M}+1)$ : 633.1413; found: 633.1400 .

UV/vis $\left(\mathrm{CHCl}_{3}\right): \lambda_{\max }(\varepsilon)=269$ (43800), $361 \mathrm{~nm}(8100)$.

\section{Metal Complex Ru-1(O)}

In an NMR tube, metal complex Ru-1 (4 mg, $3.3 \mu \mathrm{mol}, 1.0$ equiv) was dissolved in $\mathrm{MeCN}(0.75 \mathrm{~mL})$. The solution was irradiated under $\mathrm{O}_{2}$ bubbling with a $300 \mathrm{~W}$ lamp for $15 \mathrm{~min}$. The solvent was removed in vacuo to afford complex Ru-1(O); yield: $4 \mathrm{mg}$ (ca. 100\%).

IR (KBr): 3082, 2923，2853，1653，1603，1465，1447，1362, $841,764,731 \mathrm{~cm}^{-1}$.

${ }^{1} \mathrm{H}$ NMR $\left(\mathrm{CD}_{3} \mathrm{CN}\right): \delta=2.39(\mathrm{~s}, 6 \mathrm{H}), 2.62(\mathrm{~s}, 6 \mathrm{H}), 7.14(\mathrm{~d}, J=1.01$ $\mathrm{Hz}, 2 \mathrm{H}), 7.26$ (m, $2 \mathrm{H}), 7.47$ (m, $2 \mathrm{H}), 7.67(\mathrm{~m}, 2 \mathrm{H}), 7.86(\mathrm{~m}, 2 \mathrm{H})$, 7.89 (dd, $J=8.21,5.43 \mathrm{~Hz}, 2 \mathrm{H}), 8.02(\mathrm{dt}, J=7.96,1.52 \mathrm{~Hz}, 2 \mathrm{H})$, $8.12(\mathrm{dt}, J=7.89,1.39 \mathrm{~Hz}, 2 \mathrm{H}), 8.24(\mathrm{dd}, J=5.31,1.26 \mathrm{~Hz}, 2 \mathrm{H})$, $8.52(\mathrm{~d}, J=7.83 \mathrm{~Hz}, 2 \mathrm{H}), 8.55(\mathrm{~d}, J=8.34 \mathrm{~Hz}, 2 \mathrm{H}), 9.41$ (dd, $J=8.21,1.39 \mathrm{~Hz}, 2 \mathrm{H})$.

MS (ESI): $m / z=1067.13\left(\mathrm{M}^{+}-\mathrm{PF}_{6}^{-}\right)$.

HRMS: $m / z$ calcd for $\mathrm{C}_{48} \mathrm{H}_{36} \mathrm{~N}_{8} \mathrm{O}_{2} \mathrm{~S}_{2} \mathrm{Ru}\left(\mathrm{M}^{2+}-2 \mathrm{PF}_{6}\right)$ : 461.0718; found: 461.0720 .

UV/vis $(\mathrm{MeCN}): \lambda_{\max }(\varepsilon)=285$ (67400), 418 (13100), $451 \mathrm{~nm}$ (15700).

\section{Acknowledgment}

We thank the Swiss National Science Foundation for financial support.

\section{References}

(1) (a) Frimer, A. A. Chem. Rev. 1979, 79, 359. (b) Frimer, A. A. Isr. J. Chem. 1981, 21, 194. (c) Frimer, A. A. J. Photochem. 1984, 25, 211.

(2) Schweitzer, C.; Schmidt, R. Chem. Rev. 2003, 103, 1685.

(3) Vaz, C.; Griffin, G.; Christensen, S.; Lankin, D. Heterocycles 1981, 15, 1643.

(4) (a) Turner, J. A.; Herz, W. J. Org. Chem 1977, 42, 1657. (b) Jefford, C. W.; Boschung, A. F. Helv. Chim. Acta 1974, 57, 2257. (c) Politzer, I. R.; Griffin, G. W. Tetrahedron Lett. 1973, 4775.

(5) Schultz, A. G.; Schlessinger, R. H. Tetrahedron Lett. 1970, 2731. 
(6) (a) Ramiro, P.; Garcia-Fresnadillo, D.; Orellana, G. Tetrahedron 2005, 61; 9478. (b) Abdel-Shafi, A. A.; Worrall, D. R.; Ershov, A. Y. Dalton Trans. 2004, 30. (c) Abdel-Shafi, A. A.; Beer, P. D.; Mortimer, R. J.; Wilkinson, F. Helv. Chim. Acta 2001, 84, 2784. (d) Li, C.; Hoffman, M. Z. J. Phys. Chem. A 2000, 104, 5998.

(e) Abdel-Shafi, A. A.; Beer, P. D.; Mortimer, R. J.; Wilkinson, F. Phys. Chem. Chem. Phys. 2000, 2, 3137. (f) Zahir, K. O.; Haim, A. J. Photochem. Photobiol. A 1992, 63, 167. (g) Demas, J. N.; McBride, R. P.; Harris, E. W. J. Phys. Chem. 1976, 80, 2248. (h) Demas, J. N.; Diemente, D.; Harris, E. W. J. Am. Chem. Soc. 1973, 95, 6864.

(7) (a) Hartmann, P. Anal. Chem. 2000, 72, 2828.

(b) Carraway, E. R.; Demas, J. N.; DeGraff, B. A.; Bacon, J. R. Anal. Chem. 1991, 63, 337. (c) Adick, H. J.; Schmidt, R.;
Brauer, H. D. J. Photochem. Photobiol., A 1988, 45, 89. (d) Schmidt, R.; Brauer, H. D. J. Photochem. 1984, 25, 489. (e) McDonagh, A. F. Biochem. Biophy. Res. Commun. 1971, $44,1306$.

(8) Henderson, B. W.; Dougherty, T. J. Photochem. Photobiol. 1992, 55,145

(9) Griffin, G. W.; Kirschenheuter, G. P.; Vaz, C.; Umrigar, P. P.; Lakin, D. C.; Christensen, S. Tetrahedron 1985, 41, 2069.

(10) Bodige, S.; MacDonnell, F. M. Tetrahedron Lett. 1997, 38, 8159.

(11) Krayushkin, M. M.; Shirinian, V. Z.; Belenkii, L. I.; Shadronov, A. Y.; Vorontsova, L. G.; Starikova, Z. A. Russ. Chem. Bull., Int. Ed. Engl. 2002, 51, 1510.

(12) Kühni, J.; Belser, P. Org. Lett., accepted for publication. 